\title{
Joint production and major maintenance planning policies of a manufacturing system with deteriorating quality
}

\begin{abstract}
We investigate the simultaneous production planning and quality control problem for an unreliable single machine manufacturing system responding to a single product type demand. The machine is subject to deteriorations, and their effect is observed mainly on the rate of defectives, which increases continuously over time. Due to the uncertainty caused by failures, the machine may not meet long-term demand, and an overhaul can be conducted in order to counter the effect of the deterioration. The main objective of this study is to simultaneously determine the optimal production plan and overhaul schedule for the analyzed manufacturing system, in order to minimize the total cost, comprising the inventory, backlog, repair and overhaul cost, over an infinite planning horizon. A stochastic dynamic programming model is proposed, in which a numerical scheme is adopted to solve the optimality condition equations. It is observed that the optimal control policy is described by a machine deterioration-dependent hedging point policy (MDDHPP). To accurately approximate the related control parameters, a simulation optimization approach based on design of experiments, simulation modeling and response surface methodology is applied. The results obtained provide a better understanding about the influence of the deterioration of quality in the production and overhaul policies. A numerical example and an extensive sensitivity analysis are conducted, and show the robust behavior and usefulness of the policy obtained.
\end{abstract}

Keywords: Production control, Numerical Methods, Simulation, Response Surface Methodology, Manufacturing systems, Defective. 


\section{Introduction}

Production planning has been studied by several authors, with the common objective of improving productivity. However, a major limitation encountered in most of the literature in this domain lies in the assumption that all the parts produced are conforming items; an assumption which is obviously not realistic in industrial contexts. Fortunately, the inter-relation between productivity and quality has been received growing attention of researchers. We start by examining the need to integrate quality aspects in production policies, since all companies must satisfy high levels of productivity and high standards of quality. Additionally, if we take into account that manufacturing systems progressively degrade over time means that, this factor may have an impact on its operating conditions. Therefore, through this research, we contribute to a better understanding of the inter-relation between production planning and quality, in the case where the manufacturing system is subject to deterioration, which has a negative influence on the quality of parts produced.

In the literature, various authors have covered the production planning problem for unreliable manufacturing systems; for example, in the production systems studied by Kimemia and Gershwin (1983) and Akella and Kumar (1986), the control policy that they obtained was found to have a structure called hedging point policy. The importance of such a policy lies in being an efficient way of determining production policies for manufacturing systems. Following these two studies, several extensions to this research area were realized, considering a wide range of aspects such as; transportation delay from the machine to the inventory as in Mourani et al. (2008), multiple-types production satisfying a low and a high demand as in Chan et al. (2008), remanufacturing operations considering a reserve logistic network as in Kenne et al. (2012), etc. Nevertheless, the majority of such extensions on production planning have not covered the influence of quality issues on the control policy. While the importance of quality cannot be ignored, it should be noted that it reflects the need for further analysis of the inter-relation between production and quality aspects. Some papers have highlighted the importance of this inter-relation, such as that by Inman et al. (2003), which presented a comprehensive list of research issues involving the relationship between quality and production system design. However, consideration of quality issues only started growing with the series of works of Kim and Gershwin (2005, 2008), who introduced mathematical models to evaluate the performance of small and large production systems with quality and operational failures. Colledani and Tolio (2006, 2009, 2011) similarly addressed the evaluation of the performance of production systems, where the behavior of machines were monitored by control charts. Although these papers study the influence of quality aspects, their focus is on performance measures, whereas our research approach is different. We focus mainly on the structure of the control policy, and we aim to investigate the impact of quality aspects on the production control rule.

A recent area of research has emerged addressing quality issues on the production policy; Radhoui et al. (2009), for instance, used the rate of defectives as a decision variable to determine when to perform preventive maintenance and define the buffer size. The simultaneous determination of maintenance activities and production planning is covered by Njike et al. (2009), who applied several operational states that monitor the system's 
condition. They used the quantity of defective products as feedback to optimally control the system. Further, Mhada et al. (2011) analyzed the production control problem for an unreliable manufacturing system producing a random fraction of defective items, and succeeded in stating analytical expressions for the production threshold and the optimal cost. Additionally Dhouib et al. (2012) incorporates to the production planning problem, an age-dependent preventive maintenance policy that reduces the shift rate to the out of control state, where their productive systems produces non-conforming items. Despite the pertinence of these works, we conjecture that the joint production and quality control problem can be studied from a different perspective. For instance, this includes bearing in mind that in real industrial contexts, the production system is subject to deteriorations (because of an infinite set of factors), meaning therefore that the effect of the deterioration may certainly have an impact on the quality of the parts produced. This effect will allow us to extend the concept of deterioration, and relate this factor to the quality yield of the production system. We find some support for our conjecture in the area of deteriorating systems.

Many papers have been published in the area of deteriorating systems, with the typical method used to model deterioration based on the concept of imperfect maintenance. A good discussion on the subject of imperfect maintenance can be found in Pham and Wang (1996). In addition, Wang and Pham (1999) present an interesting method, and propose that after an imperfect repair, the operating time of the system decreases as the number of repairs increases. The idea to treat certain deterioration in the operating conditions was extended by Lam (2007), who proposed that the operating times after a repair decrease, while the consecutive repair times after failure increase. We find another approach for modeling deteriorating systems in the work of Dehayem et al. (2011), who described a model in which the operating time of the production system follows a decreasing function given by the age of the machine, while the repair time consists of an increasing function with the number of failures. As can be seen from the previous models, it is assumed that deterioration has an effect on the availability of the production system, and that it is used as indicator of the level of deterioration either the age of the machine or the number of failures. Nevertheless, these models do not link the degrading process to the parts quality. This observation in turn raises the question of whether it is possible to relate the deterioration phenomenon with the quality yield of the production system. Typically, an efficient alternative for determining the optimal control policies of stochastic manufacturing systems has been the use of simulation optimization approaches.

Simulation modeling has proved to be an effective means to analyze manufacturing systems, as observed in the work of Lavoie et al. (2009a), who compared different pull control mechanisms for homogenous transfer lines. Simulation has also been applied to compare different maintenance strategies, such as in Boschian et al. (2009), where they analyzed the case of two machines working in parallel, applying different maintenance strategies. Other applications of the simulation optimization approach, such as the presented by Berthaut et al. (2010), deal with the determination of production and periodic preventive maintenance rates. Recently, this hybrid methodology was extended by Gharbi et al. (2011), who analyzed the case of the production control problem of a manufacturing cell comprising a central and a reserve machine. Moreover Hajji et al. (2011) used a simulation based approach to determine the production control parameter 
and product's specification limits that have a direct influence in the rate of non-conforming items. A closer look at these models reveals that this simulation optimization approach is not yet in use in cases where the degrading process of the production system has a continuous deterioration on the parts quality.

Therefore, given this context, we intend to develop a new model for the simultaneous production and quality control policy of a mono-product manufacturing system, composed of a single unreliable machine that is subject to progressive quality deterioration and uncertainties. This is motivated by the need to study the inter-relation between quality issues and the production policy, where phenomena such as deterioration are present. Furthermore, the notion of relating the deterioration of the machine with the parts quality is based on the concept of worse repairs (a maintenance action which increases the rate of defectives). The uncertainties are due to machine failures in a dynamic continuous time stochastic context. We develop a stochastic dynamic programming model with two decision variables, the production rate and the quality decision related to the overhaul strategy, which counters the effect of the deterioration. This specific problem has not been yet addressed in the literature. The resultant control policy called Machine Deterioration Dependent Hedging Point Policy (MDDHPP), adjusts the control parameters according to the level of deterioration of the machine. The contribution of this article is further illustrated by the robust behavior of the MDDHPP facing several variations of the system parameters in a sensitivity analysis, and providing a better knowledge of the production system behavior. We propose a simulation optimization approach combined with the control theory to achieve a close approximation of the optimal control policy parameters.

The remainder of the paper is structured as follows. After an overview of the literature in section 1 , the notations and the system description are presented in section 2 . The control problem statement is detailed in section 3. Numerical methods are applied to characterize the structure of the obtained control policy in section 4. The proposed simulation optimization approach is presented in section 5, along with a detailing of the simulation model and its validation. In section 6, a numerical example is reported to illustrate the system's behavior. A sensitivity analysis of the control policy is presented in section 7, with regards to different cost parameters and trajectories of the rate of defectives. Finally, concluding remarks that illustrate new insights into this topic are given in section 8 .

\section{Notation and system description}

This section presents the notation and the system description of the manufacturing system under consideration.

\subsection{Notation}

The following notations are used:

$x(t) \quad$ Inventory level at time $t$

$u(t) \quad$ Production rate of the manufacturing system at time $t$ 


$\begin{array}{ll}n(t) & \text { Current number of failures at time } t \\ d & \text { Demand rate } \\ \xi(t) & \text { Mode of the machine at time } t \\ u_{\max } & \text { Maximum production rate } \\ \beta & \text { Rate of defectives } \\ \rho & \text { Discount rate } \\ \left.\lambda_{\alpha \alpha^{\prime} \cdot} \cdot\right) & \text { Transition rate from mode } \alpha \text { to mode } \alpha^{\prime} \\ v_{\min } & \text { Minimum overhaul transition rate } \\ v_{\max } & \text { Maximum overhaul transition rate } \\ g(\cdot) & \text { Cost rate function } \\ J(\cdot) & \text { Expected discounted cost function } \\ V(\cdot) & \text { Value function } \\ \tau & \text { A jump time of the process } \xi(t) \text { defined in the reset function } \\ c^{+} & \text {Incurred cost per unit of produced parts for positive inventory } \\ c^{-} & \text {Incurred cost per unit of produced parts for backlog } \\ c_{o} & \text { Overhaul cost } \\ c_{r} & \text { Repair cost } \\ N & \text { Maximum number of failures where the system is still operational } \\ n_{f} & \text { Number of failures needed to perform the overhaul }\end{array}$

\subsection{System description}

The production system under consideration consists of an unreliable single machine producing one part type. The block diagram of the production system is presented in Figure 1. The machine is unreliable, and is subject to random events, such as failures, and different maintenance activities, such as repairs and overhauls. We conjecture that the machine is subject to deterioration, and this phenomenon is tied directly to an increasing defective rate. The quality deterioration leads to the integration of quality related decisions, such as the overhaul strategy that counters the effect of the quality deterioration, into the model. Since the quality of the parts produced is not perfect, the product stock is composed of a mixture of flawless and defective products. In this domain, it is generally assumed that deterioration affects the availability of the system, but in this paper, however, we focus on its effect on the quality of the parts produced, and more specifically on the rate of defectives. When the machine is at failure state, a worse repair is conducted, having the particular characteristic to deteriorate the machine, thereby increasing the rate of defectives. We thus tie the number of failures to the quality deterioration; some reasons justifying this condition are discussed later on this paper. The model's decision variables are related with the production planning and the overhaul strategy. The objective of the model is to determine the simultaneous production and overhaul policies that minimize the total incurred cost, which is composed of the repair cost, the overhaul cost, the inventory cost and the backlog cost of units. 


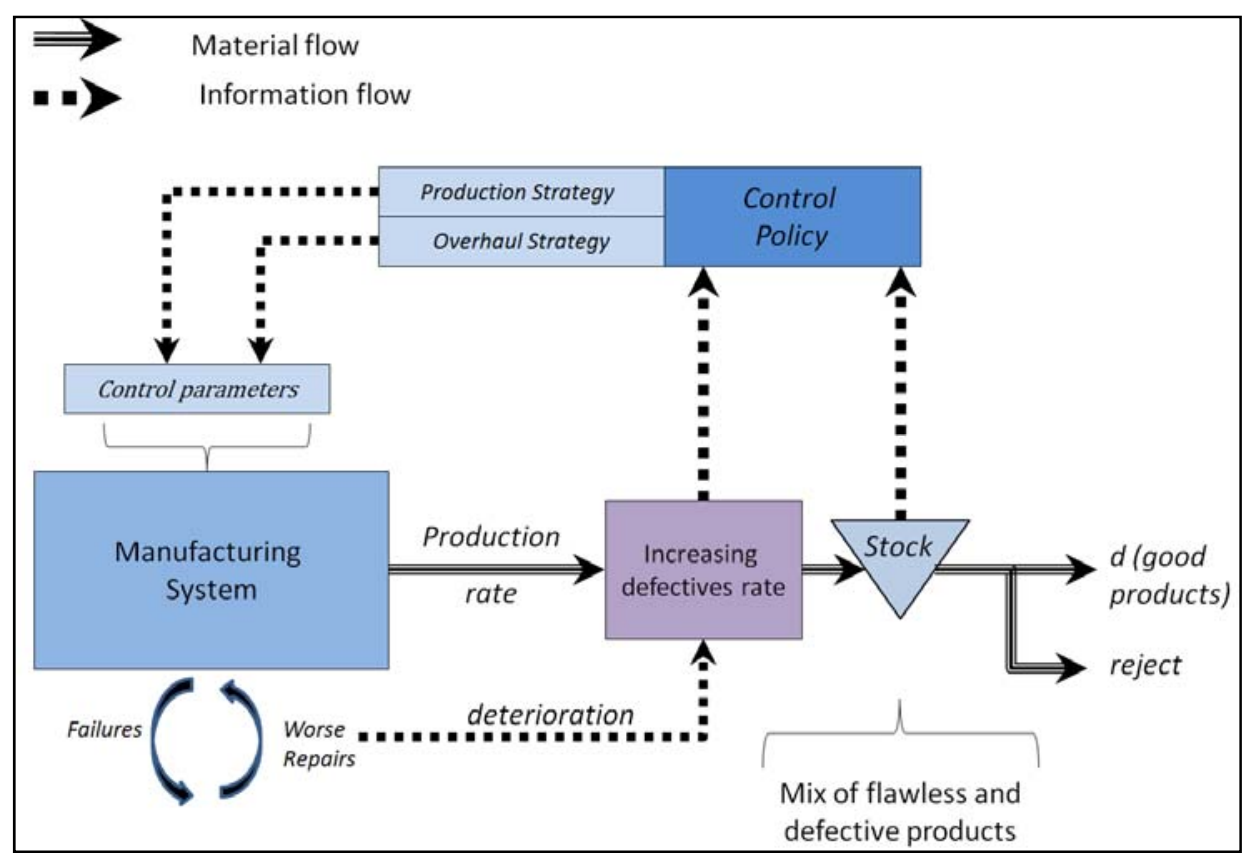

Figure 1: Block diagram of the manufacturing system under study

\section{Control problem statement}

In this section, we present the formulation of the control problem for the manufacturing system presented in section 2.2. As mentioned previously, the production system consists of a single machine that produces one type of product to meet client demand. The system is subject to random events (failures, repairs, and overhauls) as well as to quality deterioration. The machine has three modes, described by the stochastic process $\{\xi(t), t \geq 0\}$, with values in $\Omega=\{1,2,3\}$. The machine is available when operational $(\xi(t)=1)$, and unavailable when under repair $(\xi(t)=2)$ or under overhaul $(\xi(t)=3)$. The overhaul refers to a perfect maintenance whose benefit is to counter quality deterioration and restore the machine to as-good-as-new conditions.

At any given time $t$, the system is characterized by the machine mode $\xi(t)$, the number of failures $n(t)$ and the stock level $\mathrm{x}(t)$. According to standard notation, let $d$ be the demand to be satisfied and $u(t)$ the production rate at time $t$. At any time instant, the production rate $u(t)$ of the machine has to satisfy the capacity constraint: $0 \leq u(t) \leq u_{\max }$, where $u_{\max }$ is the maximum production rate.

Our primary concern in this model is that the quality of the parts produced is continuously deteriorated by the degrading process of the manufacturing system, as suggested by Colledani and Tolio (2011) and Kim and Gershwin (2008). These authors conceived the deterioration-quality relationship. To exploit this link in our model, we assume that the deterioration of the machine has the effect of increasing the rate of defectives. Hence, we propose that the dynamics of the inventory/backlog of products $x(t)$ evolves according to the following differential equation: 


$$
\dot{x}(t)=u(\cdot)-\frac{d}{(1-\beta(n))}, x(0)=x_{0}
$$

where $\beta(n)<1, x_{0}$ is the initial stock level, $n$ is the current number of failures at time $t$, $\beta(n)$ is a function of the rate of defectives, and the quantity $\frac{d}{(1-\beta(n))}$ represents the adjusted demand that includes defective products. Our remaining problem with this equation (1) is to define how exactly the function $\beta(n)$ relates the deterioration of the machine with the rate of defectives. We find some useful ideas that sketch this condition in the area of deteriorating systems.

We should recall that at failure, the maintenance option available is to conduct a worse repair, defined in Wang (2002) as the maintenance action where the system's operating conditions become worse with this sort of repair. Some reasons accounting for this deterioration is that at failure, the faulty component is only partially repaired, the influence of human errors, etc. Additionally, imperfect maintenance methods, such as those presented by Wang and Pham (1999) and Lam et al. (2004), use the number of repairs or the number of failures to define the level of deterioration of the system, this allows us to define the failures-deterioration relationship. In fact, in these models a certain trend has been observed in the deterioration, and their results have been applied successfully even to real industrial data. We therefore extend the concept of worse repairs to model quality deterioration, and based on the relationships between failures-deterioration and deterioration-quality, we propose the increasing function (2), which defines the rate of defectives as a function of the number of failures, as follows:

$$
\beta(n)=\beta_{0}+\beta_{1}\left(\frac{n}{N}\right)^{r}
$$

where $\beta_{0}$ is the value of the rate of defectives at initial conditions (normally with a very low value), $N$ is the number of failures where the system is still operational, and $\beta_{1}$ and $r$ are given parameters. It turns out that our model is a derivation of a Markov model because the transitions between modes are not affected by the failures. However, as the rate of defectives follows a defined trajectory given by equation (2), we need the number of failures $n$ in order to properly characterize the state of the system.

Before completing the problem formulation, we would like to draw the reader's attention to an important technical detail. Typically, the manufacturing system will only meet the conditions needed to fulfill the demand rate $d$ over an infinite horizon, and reach steady-state, only if the system is feasible. In other words, the production system must satisfy the following feasibility condition:

$$
u_{\max } \cdot \pi_{1} \geq d \cdot[1+\beta(n)]
$$


where, $\pi_{1}$ is the limiting probability for the operational state. Let $Q(\cdot)=\left\{\lambda_{\alpha \alpha^{\prime}}(\cdot)\right\}$ refer to the related transition matrix. Therefore $\pi_{1}$ can be computed as follows:

$$
\pi_{i} \cdot Q(\cdot)=0 \quad \text { and } \quad \sum_{i=1}^{3} \pi_{i}=1
$$

For our case of study, the solution of $\pi_{1}$ for the system of equations (4) yields to the following expression:

$$
\pi_{1}=\frac{1}{1+\frac{\lambda_{12}}{\lambda_{21}}+\frac{\lambda_{13}}{\lambda_{31}}}
$$

where $\lambda_{\alpha \alpha^{\prime}}$ are the transition rates from mode $\alpha$ to mode $\alpha^{\prime}$. In a practical sense, the value of the parameters needed by equation (2) can be determined from the analysis of maintenance service data. The advantage of equation (2) is that we can change the value of the parameter $r$ to adjust the trend of the defective rate for a specific machine. In Figure 2, we present several trajectories for the rate of defectives as an illustration, applying different values of the parameter $r$.

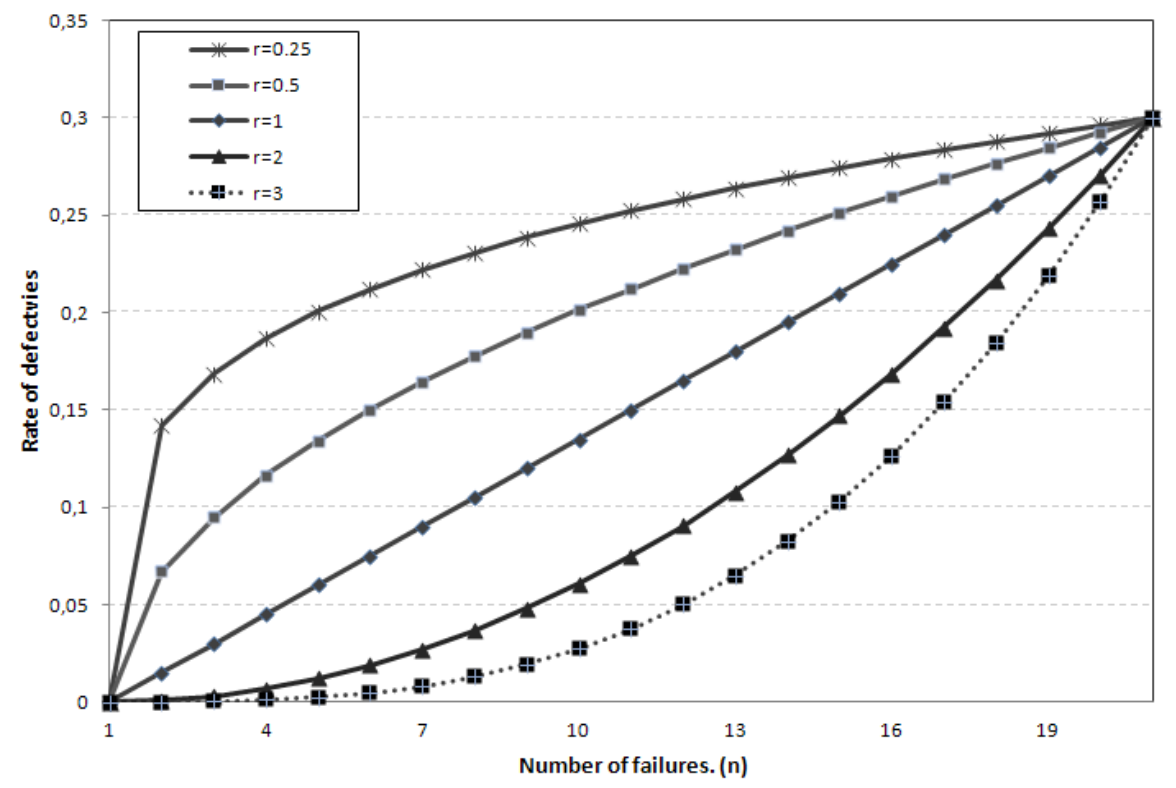

Figure 2: Trend of the rate of defectives for different values of the parameter $r$.

Returning to the model formulation, the machine's mode changes from operation mode to overhaul mode with a transition rate denoted by $\lambda_{13}=v(\cdot)$. The rate $v(\cdot)$ is assumed to be a control variable, and the decision to send the machine to overhaul is taken while the 
machine is operational. The inverse of $v(\cdot)$ represents the expected delay time between the decision to perform the overhaul and the effective switch from operation mode to overhaul mode. In other words, $\frac{1}{v(\cdot)}$ corresponds to the delay between the call of a technician and his/her arrival. Moreover, we assume that the following constraint holds for the overhaul rate:

$$
v_{\min } \leq v(\cdot) \leq v_{\max }
$$

where $v_{\min }$ and $v_{\max }$ denote the minimum and maximum overhaul rate, respectively. The instantaneous cost function of the model at mode $\alpha \in \Omega$, is defined by the following equation:

$$
g(\alpha, x, n, u, v)=c^{+} x^{+}+c^{-} x^{-}+c_{r} \cdot \operatorname{Ind}\{\xi(t)=2\}+c_{o} \cdot \operatorname{Ind}\{\xi(t)=3\}
$$

with:

$$
\begin{gathered}
x^{+}=\max (0, x) \\
x^{-}=\max (-x, 0) \\
\operatorname{Ind}\{\xi(t)=\alpha\}= \begin{cases}1 & \text { if } \xi(t)=\alpha \\
0 & \text { otherwise }\end{cases}
\end{gathered}
$$

where $c^{+}$is the inventory cost, $c^{-}$is the backlog cost, $c_{r}$ is the repair cost and $c_{o}$ is the overhaul cost. The control variables of the model are the production rate $u(\cdot)$ and the overhaul rate $v(\cdot)$; the set of admissible decisions $(u, v)$ depends on the stochastic process and, is given by:

$$
\Gamma(\alpha)=\left\{(u(\cdot), v(\cdot)) \in \mathrm{R}^{2}, \quad 0 \leq u(\alpha, \cdot) \leq u_{\max }, v_{\min } \leq v(\alpha, \cdot) \leq v_{\max }\right\}
$$

Our objective is to control the production rate $u(\cdot)$ and the overhaul rate $v(\cdot)$ in order to minimize the integral of the expected discounted cost given by:

$$
\begin{aligned}
& J(\alpha, x, n, u, v)= \\
& E\left[\int_{0}^{\infty} e^{-\rho t} g(\cdot) d t \mid \alpha(0)=\alpha, x(0)=x, n(0)=n\right], \quad \forall u(\cdot), v(\cdot) \in \Gamma(\alpha)
\end{aligned}
$$


where $\rho$ is the discounted rate and $(\alpha, x, n)$ are the initial state conditions. Optimal policies are obtained by searching in the value function:

$$
V(\alpha, x, n)=\inf _{(u, v) \in \Gamma(\alpha)} J(\alpha, x, n, u, v)
$$

The value function $V(\alpha, x, n)$ satisfies specific properties called optimality conditions. In Appendix A, it is shown that the value function $V(\cdot)$ satisfies the so-called Hamilton-Jacobi-Bellman (HJB) equations. Such equations describe the optimally conditions of the problem, in addition to determining the optimal feedback control $(u, v)$. In this case, the derivation of the optimality conditions leads to the following HJB equations:

$$
\begin{aligned}
& \rho V(\alpha, x, n)= \\
& \inf _{(u, v) \in \Gamma(\alpha)}\left\{g[\alpha, x, n, u, v]+\frac{\partial V}{\partial x}[\alpha, x, n] \dot{x}+Q(\cdot) V[\alpha, x, \varphi(\xi, n)](\alpha)\right\}
\end{aligned}
$$

where $\frac{\partial V}{\partial x}$ is the derivative of the value function. The control policy $(u, v)$ denotes a minimizer of the right-hand-side of the HJB equations, and therefore, the controls obtained are optimal. Because of the randomness of $\alpha$, the control policy is a feedback control rule based on the inventory level $x$, the mode of the machine $\alpha$, and the number of failures $n$. Furthermore, as the overhaul activity restores the rate of defectives $\beta(n)$ to initial conditions, at a jump time $\tau$ for the process $\xi(t)$, we define a reset function $\varphi(\xi, n)$ by the following relationship:

$$
\varphi(\xi, n)=\left\{\begin{array}{cc}
n+1 & \text { if } \xi\left(\tau^{+}\right)=1 \text { and } \xi\left(\tau^{-}\right)=2 \\
0 & \text { if } \xi\left(\tau^{+}\right)=1 \text { and } \xi\left(\tau^{-}\right)=3 \\
n & \text { otherwise }
\end{array}\right.
$$

We conclude this section by stating that, when the value function $V(\cdot)$ is available, an optimal control policy can be obtained from the HJB equations (11). The fact is that in general, solving the HJB equations is usually impractical, and close-form solutions are only obtained for relatively few simple models. Even finding numerical solutions for the HJB equations (11) is a challenge. Fortunately, Boukas and Haurie (1990) implemented the Kushner' method to solve such a problem in the context of production planning. In the next section, we detail the procedure for determining control policies. 


\section{Structure of optimal control policy}

To determine the optimal policy, a solution could be approximated with the HJB equations by the application of numerical methods based on the Kushner technique. The main idea of this approach is to use an approximation scheme for the gradient of the value function $V(\alpha, x, n)$. Then, a discrete function $V_{h}(\alpha, x, n)$ is used to approximate the continuous value function $V(\alpha, x, n)$, and its partial derivative $\frac{\partial V}{\partial x}(\cdot)$ can be expressed as a function of $V_{h}(\alpha, x, n)$ and the length of the finite difference interval $h$ of the variable $x$. More details about the numerical method can be consulted in Kushner and Dupuis (1992) and in Hajji et al. (2009), and references therein. Subsequently, $V_{h}(\alpha, x, n)$ is obtained by solving a discrete dynamic programming using the policy improvement technique.

Table 1 presents the value for the set of parameters used in the numerical example. From the literature of optimal control and maintenance it is customary to assume that the cost of negative inventory is much higher than the cost of positive inventory $\left(c^{-}>>c^{+}\right)$. Furthermore, it is normal that the overhaul cost is higher than the repair cost $\left(c_{o}>c_{r}\right)$. In order that the problem makes sense, the machine parameter $u_{\max }$ satisfies the condition $u_{\max }>d>0$. From a practical point of view, we can collect historical data from maintenance services to define the transitions $\lambda_{i j}$ that denote the failure and maintenance activities duration (repair and overhaul). To determine the parameters related to the degradation of quality $\left(\beta_{0}, \beta_{1}, \mathrm{r}, \mathrm{N}\right)$, it can be consulted historical production data and fit equation (2). Additionally, as mentioned previously in section 3, for the problem to be feasible, condition (3) must be fulfilled. Without loss of generality data of Table 1 satisfies the feasibility condition until the $20^{\text {th }}$ failure.

\begin{tabular}{lcccccc}
\hline Parameter & $\mathrm{u}_{\max }($ units $/ \mathrm{hr})$ & $\mathrm{d}$ (units/hr) & $\mathrm{h}$ & $\rho$ & $\mathrm{c}+(\$ / \mathrm{units} / \mathrm{hr})$ & $\mathrm{c}-(\$ / \mathrm{units} / \mathrm{hr})$ \\
Value & 5 & 3 & 0.5 & 0.9 & 5 & 250 \\
\hline Parameter & $\mathrm{c}_{\mathrm{r}}(\$)$ & $\mathrm{c}_{\mathrm{o}}(\$)$ & $\mathrm{N}$ & $\mathrm{r}$ & $\beta_{0}$ & $\beta_{1}$ \\
Value & 5 & 10 & 20 & 1 & 0 & 0.35 \\
\hline Parameter & $\lambda_{12}(1 / \mathrm{hr})$ & $\lambda_{21}(1 / \mathrm{hr})$ & $\lambda_{31}(1 / \mathrm{hr})$ & $v_{\min }(1 / \mathrm{hr})$ & $v_{\max }(1 / \mathrm{hr})$ & \\
Value & 0.1 & 2 & 0.6 & 0.0001 & 20 & \\
\hline
\end{tabular}

Table 1: Parameters of the numerical example

It follows from the HJB equations (11) and their corresponding solution using the data of Table 1 that we obtain the control policy presented in Figure 3. The results obtained for the production policy are presented in Figure 3a, and for the overhaul policy in Figure $3 \mathrm{~b}$. Based on these graphics, it is apparent that the optimal production control policy consists of three rules, where the production rate is set to $u_{\max }, d$ and 0 , respectively. Moreover, the overhaul policy divides the plan $(x, n)$ into two regions, where the overhaul rate is set to $v_{\min }$ and $v_{\max }$. 
The optimal production threshold level is denoted by $Z p(\cdot)$, and defines the limits of the production region. The production control policy obtained is an extension of the hedging point policy, given that it respects the structure presented in Akella and Kumar (1986).

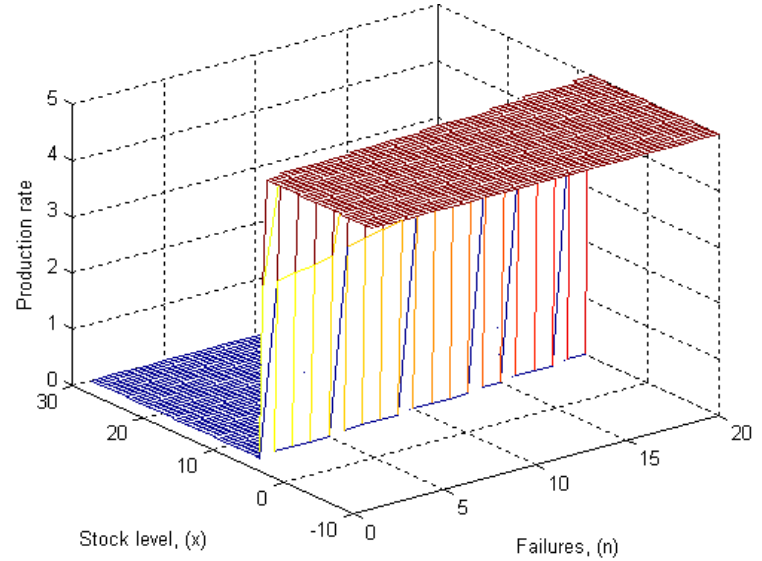

a) Production policy

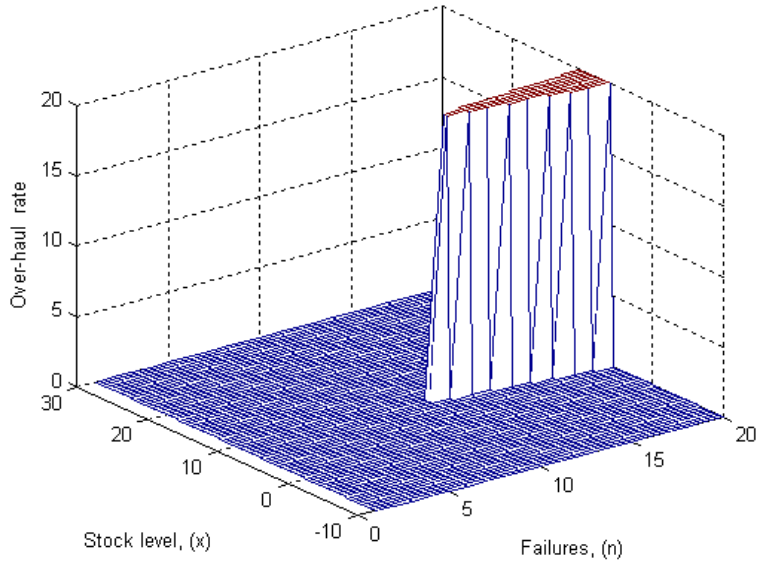

b) Overhaul policy

Figure 3: Obtained control policies

In this case due to the effect of the deterioration of the parts quality; the production policy leads to the following Machine Deterioration Dependent Hedging Point Policy (MDDHPP):

$$
u(\alpha, x, n)^{*}=\left\{\begin{array}{cc}
u_{\max } & \text { if } x(t)<Z p(n) \\
d & x(t)=Z p(n) \\
0 & x(t)>Z p(n)
\end{array}\right.
$$

where $Z p(\cdot)$ is the funtion that gives the optimal production threshold for each failure $n$. We note that the production threshold $Z p(\cdot)$ of Figure $3 \mathrm{a}$, increases progressively; this trend shows the effect of the quality deterioration on the production policy.

The overhaul policy is presented in Figure 3b. Its analysis is facilitated with the use of its boundary $B_{n}(\cdot)$. We note that Figure $3 \mathrm{~b}$ identifies two zones in the computational domain delimited by the boundary $B_{n}(\cdot)$, as follows:

- Zone $A$ : in this zone the quality deterioration, denoted by the number of failures, has a high level, which justifies the cost of performing an overhaul, and thus the overhaul rate is set to its maximum value, (i.e., $v(\cdot)=v_{\max }$ ).

- Zone $B$ : here the quality deterioration is low, meaning that an overhaul is not recommended, and the related decision variable is set to its minimum value, (i.e., $\left.v(\cdot)=v_{\text {min }}\right)$. 
To define the overhaul policy, we will now simultaneously consider the production and the overhaul boundaries as presented in Figure 4. Apparently the overhaul trace $B_{n}(\cdot)$ is

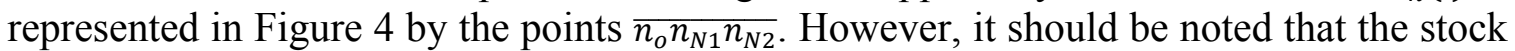
level is limited by the production threshold $Z p(\cdot)$, identified by the segment $\overline{Z p_{o} Z p_{N}}$. This observation help us to realize that only a part of the overhaul zone $A$ is used, and from what follows, this feasible overhaul region is denoted as zone $A^{\prime}$. This reduction is important as it indicates that we can define the overhaul policy knowing the point $n_{f}^{*}$, which is where the segments $\overline{Z p_{o} Z p_{N}}$. and $\overline{n_{o} n_{N 2}}$, intersect.

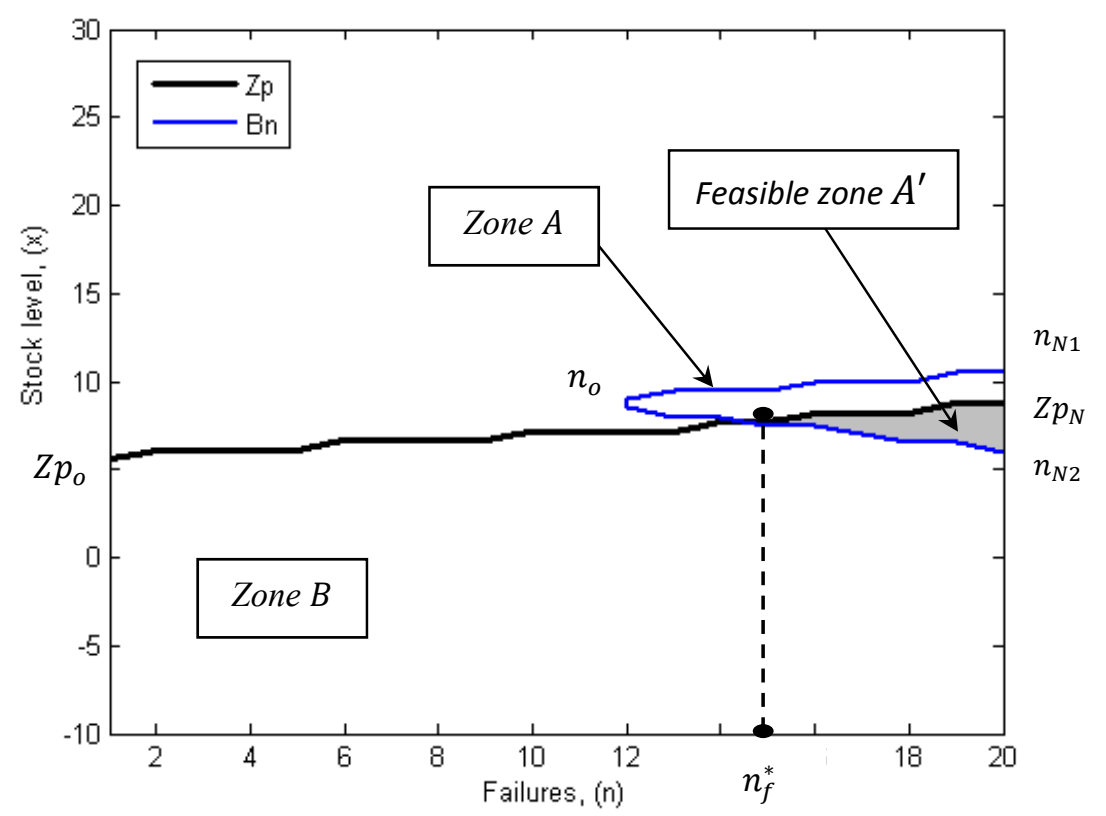

Figure 4: Intersection of the production and the overhaul trace

According to the results provided by Figure $3 b$, it is clear that the overhaul policy has a bang-bang structure. Moreover, the overhaul activity is triggered according to the policy described in Figure 4, which indicates that the overhaul activity should be performed at rate $v(\cdot)$, with:

$$
v(\alpha, x, n)^{*}=\left\{\begin{array}{cc}
v_{\max } & \text { if } n(t) \geq n_{f}^{*} \text { and } x(\cdot) \in \text { zone } A^{\prime} \\
v_{\min } & \text { otherwise }
\end{array}\right.
$$

where $n_{f}^{*}$ is the number of failures limit required to conduct the overhaul. For a given number of failures $n$, the parameter $n_{f}^{*}$ is provided by Figure 4 , as the point where the production threshold $Z p(\cdot)$ intersects the overhaul zone $A$. On the basis of the previous result, it can be observed that when the number of failures increases, the deterioration of 
the parts quality also increases, meaning that the machine is sent to overhaul more often. This highlights the fact that the production and overhaul policies are influenced by the quality-deterioration phenomenon. Summing up, we can illustrate the joint production and overhaul policies by equations (13)-(14), which are characterized by the control parameters $\left(Z p, n_{f}\right)$, where $Z p$ corresponds to the value of $Z p(\cdot)$ for each $n$ value in Figure 4.

Briefly, even though the application of the numerical methods provides the structure of the optimal control policies, the problem is that a satisfactory approximation of the control parameters would be too time-consuming to be applicable at the operational level. This is observed because the accuracy of the numerical results depends on the size of the discrete grid step $h$, as discussed in Kenné et al. (2003). To overcome this condition in the next section, we propose an alternative simulation optimization approach, to approximate the optimal control parameters $\left(Z p, n_{f}\right)$, and determine the optimal cost. The technical advantage of the simulation optimization is that it is more flexible, and allows us to examine the control policy in a wide range of time and different cost variations.

\section{Simulation optimization approach}

This section presents a simulation optimization approach having the advantage of being applicable at an operational level. The proposed control approach combines analytical and simulation models with statistical analysis, and is based on the works of Gharbi and Kenné (2000) and Berthaut et al. (2010). The block diagram of the proposed approach is presented in Figure 5, and consists of the following sequential steps:

- Mathematical formulation of the optimization problem and numerical resolution: This step consists of the representation of the simultaneous production planning and the overhaul scheduling problem through an optimal control model. The objective is to determine the control variables $(u, v)$ that minimize the incurred cost. Numerical methods are applied to determine the structure of the control policy, in which we identify the control parameters $\left(Z p, n_{f}\right)$, as discussed in section 3 and section 4 .

- Development of the simulation model: A simulation model is developed that uses the control parameters $\left(Z p, n_{f}\right)$ as inputs for conducting several simulations runs. This simulation model accurately reproduces the behavior of the manufacturing system and provides a measure of its performance, denoted by the total incurred cost. A detailed description of the simulation model is presented in section 5.1.

- Statistical analysis: Data from several simulation runs is collected to perform a design of experiments with the purpose of determining the effects of the main factors and their interactions on the total incurred cost. The design of experiments analyzes the factors with a minimal set of simulation runs. 
- Parameter Optimization: The response surface methodology is used to express a relationship between the incurred cost and the significant main factors and interactions identified. The resulted expression is then optimized to determine the best values $\left(Z p^{*}, n_{f}^{*}\right)$ of the control parameters.

- Near-optimal control policy: Finally $\tilde{u}\left(\alpha, x, n, Z p^{*}\right)$ and $\tilde{v}\left(\alpha, x, n, n_{f}^{*}\right)$ define the control policy to be applied to the manufacturing system. The application of the proposed control approach defines the production and overhaul rates described by equations (13) and (14) for the best values $\left(Z p^{*}, n_{f}^{*}\right)$ of the control factors.

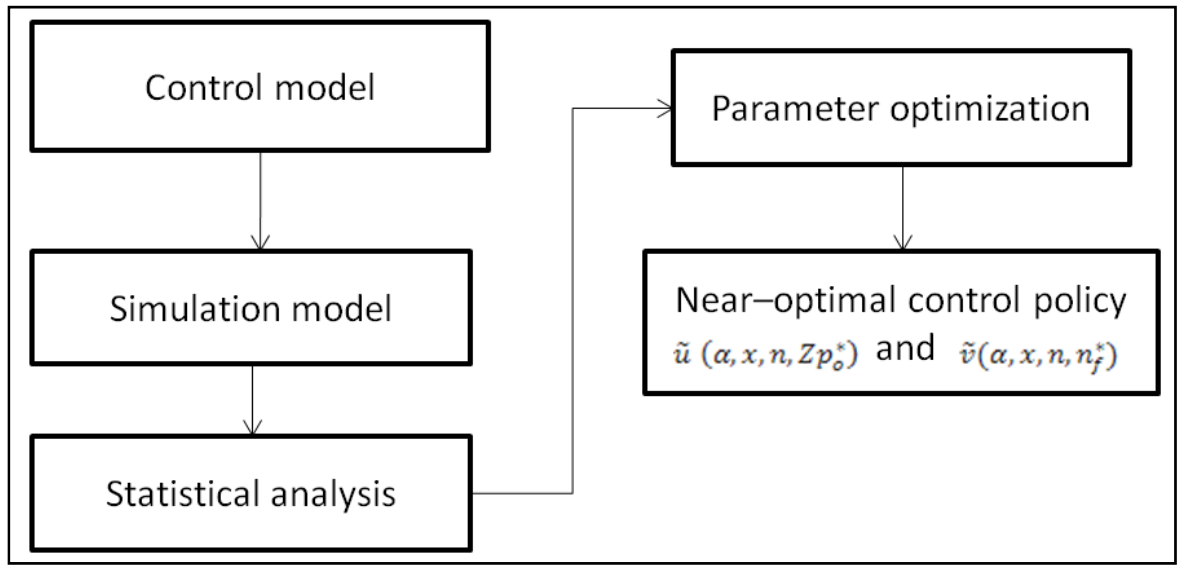

Figure 5: Proposed simulation-based control approach

\subsection{Simulation model}

A discrete/continuous simulation model was developed for the manufacturing system under analysis, and the simulation software Arena with $\mathrm{C}$ subroutines was applied. The model consisted of several networks and user routines, each of which describes a specific task or event in the system. The block diagram of the simulation model is presented in Figure 6, and its description is detailed below:

1. The INITIALIZATION block sets the values of several parameters, such as the input factors $\left(Z p, n_{f}\right)$, the demand rate $d$, the maximum production rate $u_{\max }$, and the value of the transitions for the different modes. It also defines the step specifications for the time-persistent statistics of the cumulative variables, as well as the simulation time $T_{\text {end }}$ and the length of the warm-up period.

2. The FAILURES AND REPAIRS block samples the time to failure and time to repair of the machine from their respective probability distributions. It communicates with the state equation block (5) to indicate the operational and the breakdown state; a direct communication also exists with the rate of defectives block (4) to adjust the current rate $\beta$ with every repair, and subsequently update the production threshold level. 
3. The OVERHAUL POLICY block, together with observations networks, establishes when to perform the overhaul, as defined in equation (14). It communicates with the failures-repairs block (2) and the state equation block (5) to properly synchronize different events, such as failures, repairs and overhaul. Moreover, it interacts with the rate of defectives block (4) to indicate when an overhaul has been conducted.

4. The RATE OF DEFECTIVES block receives information from the failures-repairs block (2) and the overhaul policy block (3) to correctly update the rate of defectives. Generally, at failure, the rate $\beta$, increases as defined in expression (2). Conversely, these parameters are restored to initial conditions when an overhaul is performed.

5. The STATE EQUATION block defines, in a $\mathrm{C}$ language insert, the system dynamics of the production system, which in this case, is the evolution of the inventory level denoted by the differential equation (1). For proper operation this block requires the production rate set by the production policy block (6), the rate of defectives given in block (4) and the state of the machine defined in the failures-repairs block (2) and the overhaul policy block (3).

6. The PRODUCTION POLICY block uses equation (13) to set the proper production rate comparing the current stock level with a pre-determined threshold. When the current stock level crosses the production threshold, a flag is noticed by detection mechanisms. The production rate is then adjusted and used in the differential state equation of block (5).

7. The ADVANCE TIME block updates the current time based on a combination of discrete event scheduling (consisting of failures, repairs and overhauls), continuous variables threshold crossing events and time step specifications.

8. The UPDATE STOCK LEVEL block traces any variations of the inventory level for the chosen time step. As well, it integrates the cumulative variables using the Runge-Kutta-Fehlberg algorithm.

9. At the end of the simulation time $T_{\text {end }}$, the OUTPUT block provides time persistent statistics of the positive and negative stock, the simulation length, the number of repairs and overhauls conducted, and the number of failure $n_{f}$, that is where the production threshold intersects the overhaul zone $A$. Subsequently, the total incurred cost is calculated based on the information provided by this block.

The simulation ends when the current simulation time $T_{\text {now }}$ reaches the defined simulation period $T_{\text {end }}$, which is defined as the required time to ensure steady-state conditions. The simulation model reproduces the characteristic features of the proposed manufacturing system, and a more detailed discussion about the validity of the simulation results is provided in the next section. 


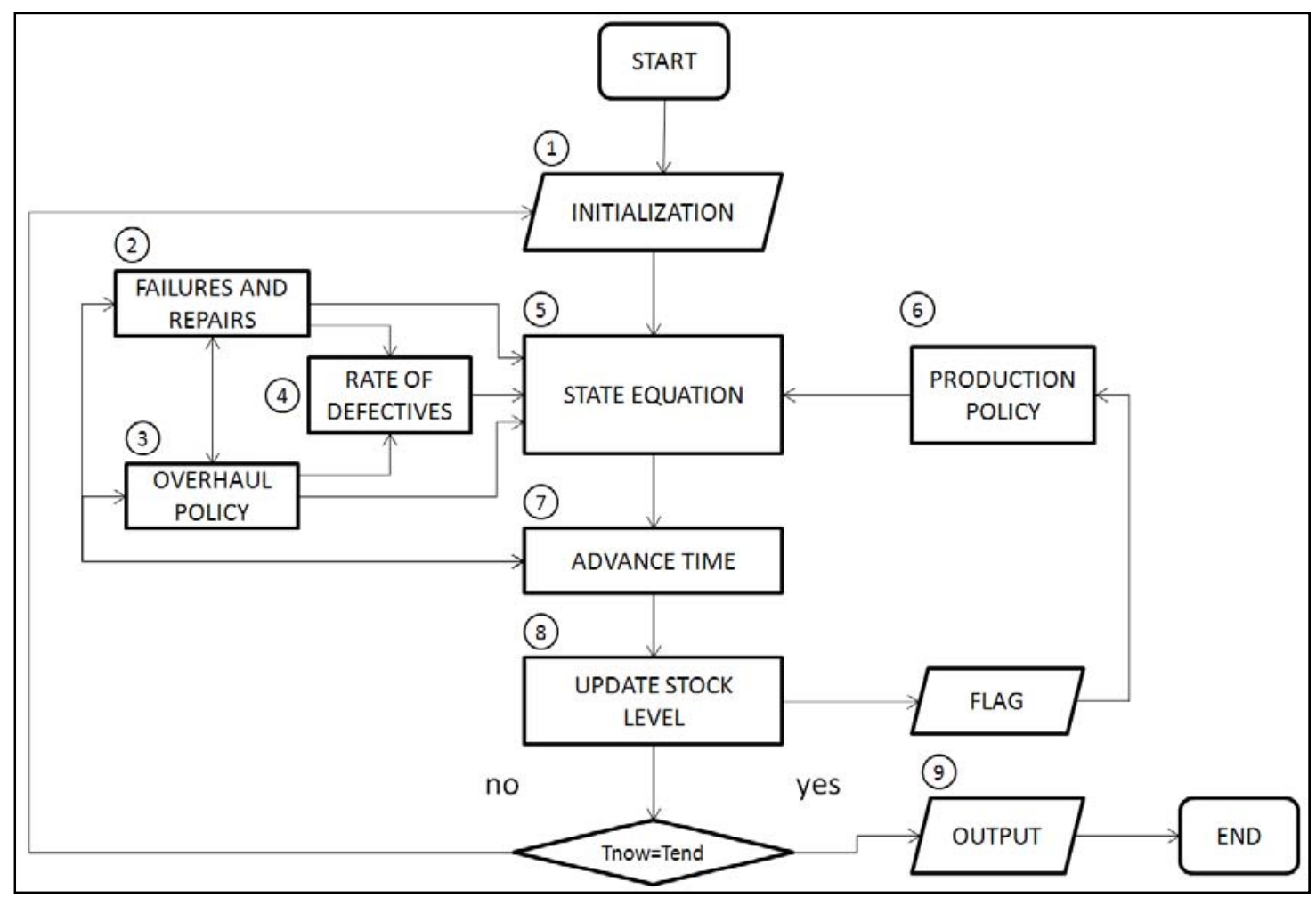

Figure 6: Simulation model block diagram

\subsection{Validation of the simulation model}

To better to verify the accuracy of the simulation model, we examine the evolution of the trajectory of the stock level. This analysis is intended to graphically evaluate whether the simulation model works according to the control policy obtained and the series of proposed assumptions discussed previously. In that regard, Figure 7 illustrates the trajectory of the inventory level $x(t)$ when the control parameters are set to $Z p_{o}=20$, and $n_{f}=11$. The evolution of the inventory level is as follows: at time $t=0$, the machine maintains a stock level denoted by the production threshold $Z p_{o}=20$, and then it experiences a series of random failures, and after every failure, the production threshold increases by a certain amount. At time $t=114$, the machine experiences its $11^{\text {th }}$ failure, and at this point the increase in the stock level is notable, as compared to the initial conditions; this point also indicates the moment to carry out an overhaul. At time $t=122.5$, after an overhaul is conducted, the rate of defectives and the production threshold are restored to initial conditions. From this point, the machine deteriorates once again, increasing the production threshold with every failure, until at time $t=233.5$, where it accumulates eleven other failures. At time $t=242$, after a second overhaul is conducted, the machine is restored to initial conditions. From this point the production system continues its normal deterioration until $t=300$, where the simulation run is completed. 


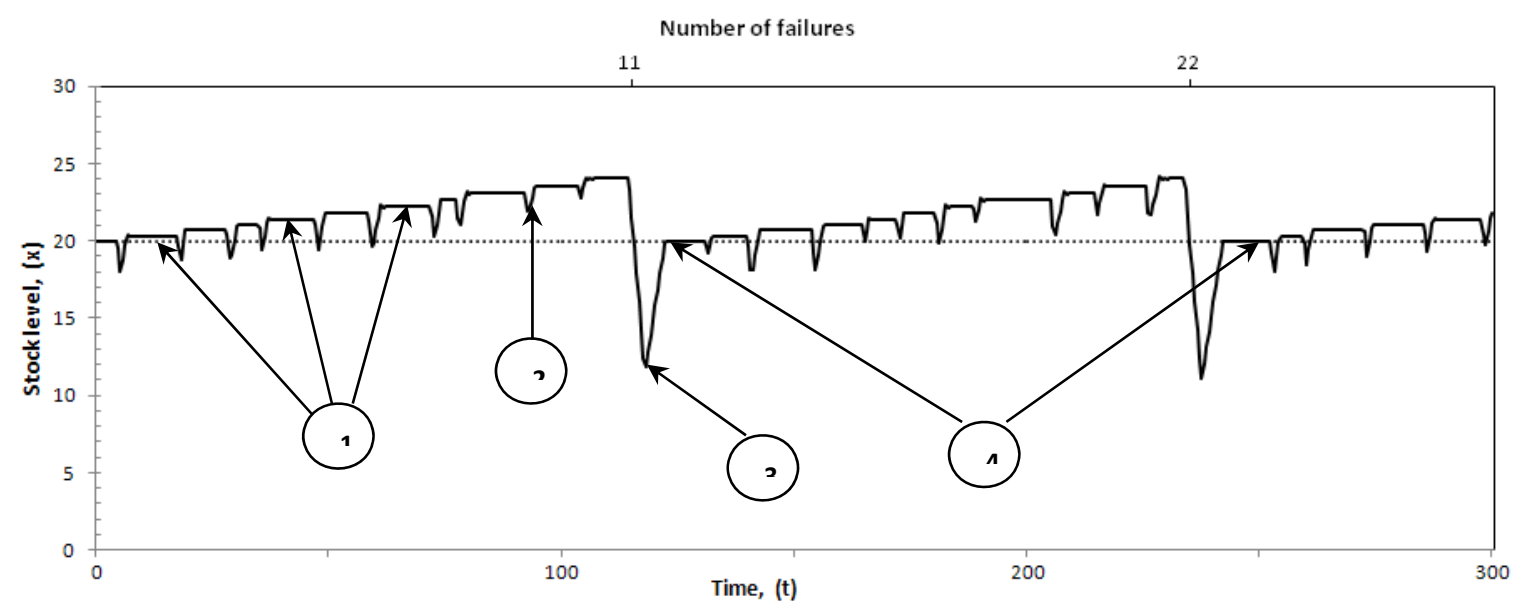

Figure 7: Stock trajectory, for $Z p_{o}=20$ and $n_{f}=11$

Summing up, from the stock trajectory of Figure 7, we note that: i) The inventory level reaches $Z p_{o}$ and this threshold, as well as the rate of defectives, increases with every failure because of the quality deterioration phenomenon, and arrows (1) illustrates the increases in the production threshold. ii) The stock level decreases during repair as indicated by arrow (2), and decreases even more when an overhaul is carried out, as indicated by arrow (3), because an overhaul requires much more time. iii) As expected, every eleven failures, an overhaul is conducted, and this activity restores the rate of defectives and the production threshold to initial conditions, as presented in arrows (4). Based on these observations, we can state that our simulation model works well, according to the assumptions of the proposed manufacturing system, and that it properly reproduces the dynamics of the stock level.

\section{Numerical example}

The determination of the control parameters for the joint production and overhaul policy is illustrated with a numerical example. We combine the simulation model with statistical analysis based on designs of experiments, and parameter optimization applying the response surface methodology. In this section, we detailed the procedure for simultaneously and efficiently varying the input variables $\left(Z p, n_{f}\right)$ of the simulation model. The goal is to identify the significant main factors and interactions that have a significant effect on the total incurred cost. Then eventually, we determine the best values $\left(Z_{p}^{*}, n_{f}^{*}\right)$ of the control parameters and calculate their respective incurred costs.

One point should be noted concerning the control parameters. It is clear from the last section that with each failure, a worse repair is conducted, which leads to different system dynamics, and that implies an increase of the production threshold and the rate of defectives. To determine the control factors, we must first define a limit for the number of failures. Let us, for instances, set $N=20$, as discussed previously in section 3 . Normally this leads to the definition of 22 parameters, with 21 related with the production threshold $Z p$ and one with the number of failures $n_{f}$. Given the convexity 
property of the value function (10), and given that an optimal solution of the control problem exists, we can define three levels for each control parameter to obtain a convex estimated cost function. In particular, we consider a second-order model to fit the cost function, and there are various possibilities for addressing this problem. For instance, a complete $3^{n}$ factorial design may be conducted, which in the case concerning 22 parameters, leads to a $3^{22}$ design. If we replicate this design four times, then altogether we will need $\left(3^{22} \times 4\right)=1.23 \times 10^{11}$ runs, which is a considerable number of calculations. The number of runs could possibly be reduced with a fractional design such as the central composite design (CCD), as reported in Montgomery (2009) and Lavoie et al. (2009b), which in the case of 22 parameters gives a total of $\left(2^{22}+2(22)+3\right) 4=1.67 \times 10^{7}$ runs. The problem is that even in this last scenario, the computational effort needed to perform the calculations would be too time-demanding. Faced with such a situation, it should first be ascertained that each of the designs presented is insurmountable, due to time and resource constraints, and mainly because a single run of our simulation model takes 4.6 seconds on average, and an exhaustive simulation of all the combinations would simply be impossible. In average $1.84 \times 10^{4}$ years would be needed to complete the runs with the $3^{22}$ factorial design, while the CCD design would require 895 days. If the number of parameters to optimize were smaller, the number of runs could considerably be reduced with a Latin Hypercube Sampling (LHS). However, this is not the case, since we have to optimize 22 parameters, and LHS is impractical when the dimensionality is too high (i.e., more than ten parameters), as suggested in Huang et al (2006). Therefore, we have to conceive alternative procedures to cope with such limitations.

To tackle this difficulty, we propose a drastic reduction in the number of simulation runs required, while simultaneously maintaining high accuracy in the results. The reduction is based on the observation that the production threshold follows a defined trajectory along the deterioration process. Hence, it is possible to define an analytical expression for the production threshold, when the manufacturing system produces a random fraction of defective products.

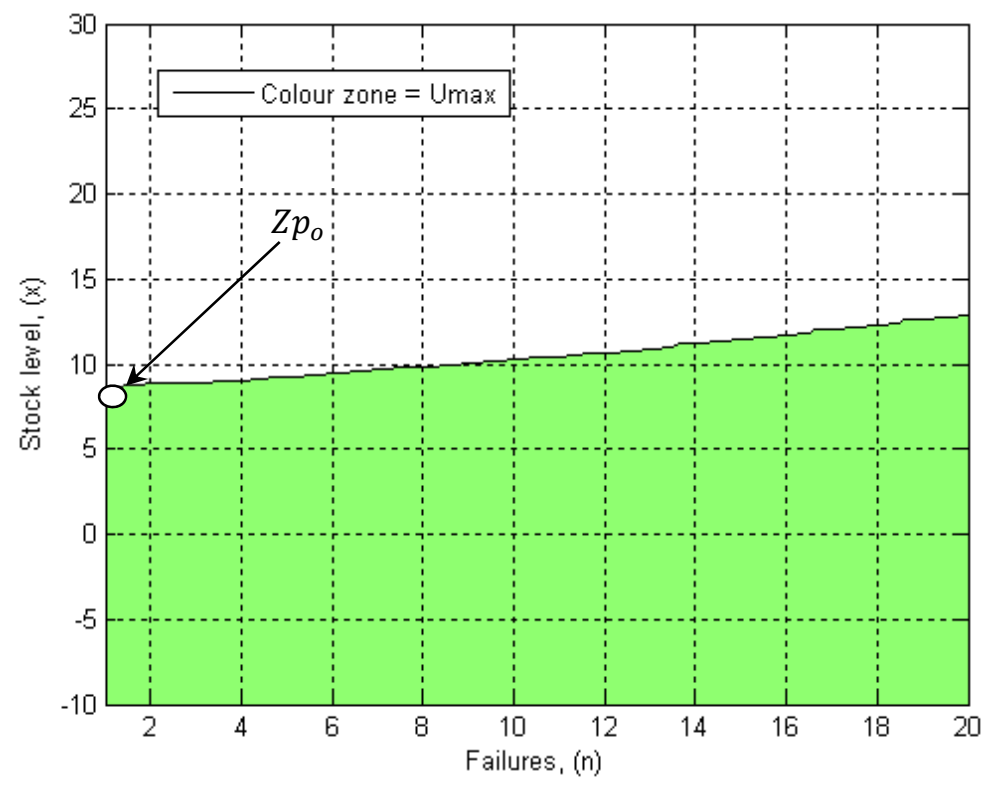

Figure 8: Trajectory of the production threshold $Z_{p}$ as a function of the number of failures $n$ 
Figure 8 depicts a trace of the production policy as a function of the number of failures. We conjecture that the trajectory of the production threshold $Z p(\cdot)$ for any number of failures can be determined by only one parameter $Z p_{o}$ which denotes the value of the production threshold before the first failure. From an adaptation of the results of Mhada et al. (2011), we can define the whole trajectory of the production threshold $Z p(\cdot)$ according to the following expression:

$$
Z p(n)=\left\{\begin{array}{cc}
\frac{Z p_{o}}{\left(1-\left[\beta(n)-\beta_{o}\right]\right)} & \text { if } 0 \leq n \leq N \\
0 & \text { otherwise }
\end{array}\right.
$$

where $Z p_{o}$ is the optimal production threshold before the first failure, $\beta(n)$ is the rate of defectives denoted by equation (2) for a given number of failures $n$, and $\beta_{o}$ is the value of the rate of defectives at initial conditions. The application of equation (15) considerably facilitates the determination of the control parameters, since adaptations of this expression can also be applied to define the feasible overhaul zone, based on the control policy discussed in section 2. Consequently, the original problem concerning 22 parameters reduces to the determination of only two factors $\left(Z p_{o}, n_{f}\right)$, leading to the possibility of applying a $3^{2}$ factorial design. Replicating this design four times implies a total of $\left(3^{2} x 4\right)=36$ simulation runs, which is much easier to compute, as compared with the complete and fractional designs. The practical advantage of equation (15) is remarkable, and in fact, modifications of this equation are implemented in our simulation model to determine the increase of the production threshold and the size of the overhaul zone.

\subsection{Statistical analysis}

The statistical analysis of the simulation data consists in conducting a multifactor analysis of variance (ANOVA). In this section, we determine the control factors $\left(Z p, n_{f}\right)$ using two independent variables $\left(Z p_{o}, n_{o}\right)$, where $Z p_{o}$ denotes the production threshold before the first failure as observed in Figure 8, and $n_{o}$ is the number of failures that represents the origin of the overhaul zone $A$, as shown in Figure 4. We identify one dependent variable denoted by the total incurred cost. As discussed previously, a complete experimental $3^{2}$ design is selected to fit the cost function, where each combination of factors is replicated 4 times, requiring 36 simulations runs in total. From off-line simulations, the replication length for each simulation run is set to $1,000,000$ time units to ensure that steady-state conditions are achieved. The cost values presented in Table 2 are considered in the statistical analysis, and the remaining parameters are defined as indicated in Table 1.

\begin{tabular}{lcccc}
\hline Parameter & $\mathrm{c}+(\$ /$ units $/ \mathrm{hr})$ & $\mathrm{c}-(\$ /$ units $/ \mathrm{hr})$ & $\mathrm{c}_{\mathrm{r}}(\$)$ & $\mathrm{c}_{\mathrm{o}}(\$)$ \\
Value & 4 & 250 & 1000 & 3000 \\
\hline
\end{tabular}

Table 2: Cost parameters for the statistical analysis 
The ANOVA is performed on the $3^{2}$ experimental design, using the statistical software STATGRAPHICS, in a bid to quantify the effects of the main factors, their interactions and their quadratic effects on the total incurred cost. Based on off-line simulation runs, we select the minimum and the maximum values of the factors $\left(Z p_{o}, n_{o}\right)$, as presented in Table 3 .

\begin{tabular}{ccccc}
\hline Factor & Low level & Center & High level & Description \\
\hline$Z p_{o}$ & 4 & 8 & 12 & Production threshold at the first failure \\
$n_{o}$ & 7 & 10 & 13 & Failure of origin of the overhaul zone $A$ \\
\hline
\end{tabular}

Table 3: Level of the independent variables

The ANOVA table corresponding to the generated data states that at a confidence level of $95 \%$, all the $p$-values are less than $5 \%$. The significant factors are identified in Figure 9, where the standardized Pareto plot is presented. The analysis also provides the proportion of the observed variability explained by the model that is denoted by the adjusted coefficient of determination $R^{2}$. In this case, we found that the model explains $94.21 \%$ of the variability observed in the incurred cost.

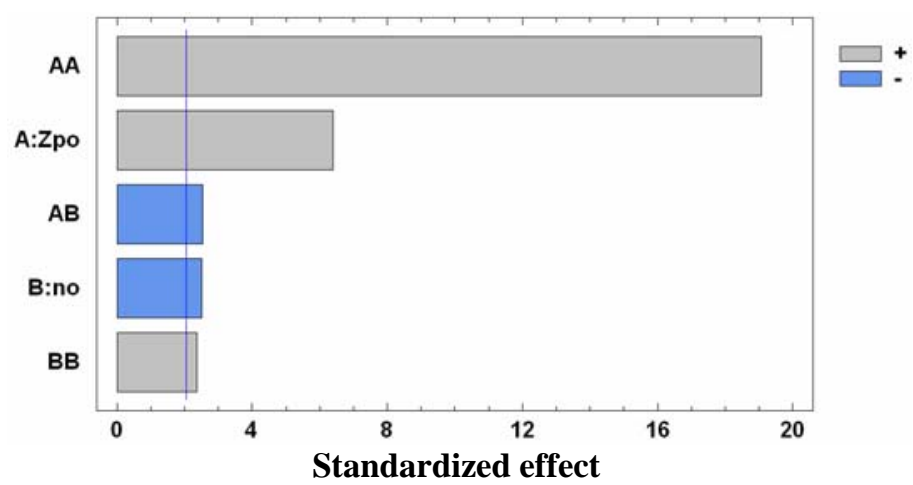

Figure 9: Standardized Pareto Plot for the total cost

Based on the ANOVA results, we found that the two main factors, $Z p_{o}$ and $n_{o}$, the quadratic effect $Z p_{o}^{2}$ and $n_{o}^{2}$ and their interaction $Z p_{o} n_{o}$ must be included in the parameter optimization since they are significant at a confidence level of $95 \%$. Moreover, the residual versus the predicted valued plot and the normality probability plot were used to test the homogeneity of the variances and the residual normality, respectively. We conclude that the total cost can be determined well by the proposed second-order model.

\subsection{Parameter optimization}

A response surface methodology is applied in order to minimize the incurred cost as a function of the significant variables identified in the last section. To this end, we assume that there exists a continuous function $\Phi$, called the response surface, which defines the total incurred cost corresponding to any given combination of the parameters $Z p_{o}$ and $n_{o}$. In this case, the second-order model obtained is given by: 
$\Phi\left(Z p_{o}, n_{o}\right)=$

$$
\begin{aligned}
& 194.243-7.3398 \cdot Z p_{o}-1.99834 \cdot n_{o}+0.525489 \cdot Z_{p}^{2}-0.0660525 \cdot Z p_{o} n_{o}+ \\
& 0.115667 n_{o}^{2}+\epsilon
\end{aligned}
$$

The projection of the cost response surface in a two-dimensional plan is presented in Figure 10. The minimum total cost is 155.29 , and is located at $Z p_{o}^{*}=7.66$ and $n_{o}^{*}=10.82$. With these values, we can determine the missing control parameter $n_{f}^{*}$ as provided bellow. In the last section, we stated that from the initial value $Z p_{o}^{*}$, the production threshold follows a defined trajectory denoted by equation (15). Moreover, from point $n_{o}^{*}$ the overhaul zone spreads in the plan intersecting the production threshold, as presented in section 4 . We postulate that a part of the overhaul trace follows an inverse trajectory of the production threshold, as observed in Figure 4, and therefore, we use a variation of expression (15) (with an inverse trend) and expression (15), (both expressions incorporated in the simulation model) to define their intersection, and determine the parameter $n_{f}^{*}$. Following this procedure, we identify the intersection of the production threshold and the overhaul zone at failure $n_{f}^{*}=13.84$. Therefore, the values $Z p_{o}^{*}=7.66$ and $n_{f}^{*}=14$ represent the best obtained parameters that should be applied in the simultaneous production and overhaul control policy.

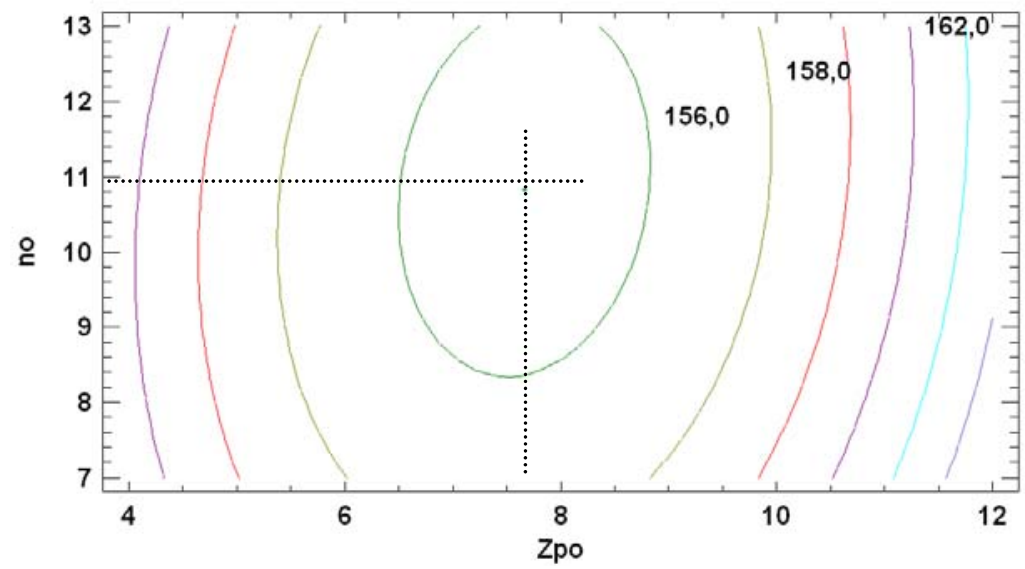

Figure 10: Contours of the estimated response surface.

If we round the number of failures to the closest integer $n_{o}^{*}=11$ and minimize the cost function (16), the optimal production threshold is $Z p_{o}^{*}=7.68$, and these parameters lead to a total cost of 155.30 . To cross-check the validity of the results obtained, we use these values as input to the simulation model to obtain a $95 \%$ confidence interval for the total cost, which in this case, is defined by:

$$
\bar{X}(y) \pm t_{n-1,1-\left(\frac{\alpha}{2}\right)} \sqrt{\frac{S^{2}(y)}{y}}=[154.89 ; 155.70]
$$

The interval was calculated with $y=35$ extra replications of the simulation model. This result confirms that our simulation optimization approach determines the optimal values of the control parameters with high precision, since the minimum cost given by the cost 
function (16) falls inside the confidence interval. With the optimal values of the independent variables, the cost is minimized and the corresponding control policy can subsequently be defined.

\section{Sensitivity analysis}

The sensitivity of the control policy obtained for a set of numerical examples is analysed with respect to different cost variations of the inventory, backlog, repair and overhaul costs (i.e., $c^{+}, c^{-}, c_{r}, c_{o}$ ). The objective of the analysis is to compare the incurred cost and control parameters for different cost scenarios derived from a basic case. Also, the effect of the variation in the trajectory of the rate of defectives on the control parameters $\left(Z p_{o}, n_{f}\right)$ is analyzed.

\subsection{Effect of the cost variation}

The goal of this analysis is to demonstrate the efficiency and robustness of our simulation optimization approach, and study the behaviour of the control policy obtained, when varying cost parameters such as: $c^{+}, c^{-}, c_{r}, c_{o}$. Thus, Table 4 illustrates eight different configurations of cost parameters, derived from a basic case by changing them to higher and lower values. These different configurations are related with variations of the inventory, backlog, repair and overhaul cost.

\begin{tabular}{|c|c|c|c|c|}
\hline Case & $c^{+}$ & $c^{-}$ & $c_{r}$ & $c_{O}$ \\
\hline Basic case & 3 & 250 & 1000 & 3000 \\
\hline \multicolumn{5}{|c|}{ Sensitivity of positive inventory cost } \\
\hline 1 & 2 & 250 & 1000 & 3000 \\
\hline 2 & 4 & 250 & 1000 & 3000 \\
\hline \multicolumn{5}{|c|}{ Sensitivity of backlog stock cost } \\
\hline 3 & 3 & 150 & 1000 & 3000 \\
\hline 4 & 3 & 350 & 1000 & 3000 \\
\hline \multicolumn{5}{|c|}{ Sensitivity of repair cost } \\
\hline 5 & 3 & 250 & 500 & 3000 \\
\hline 6 & 3 & 250 & 1500 & 3000 \\
\hline \multicolumn{5}{|c|}{ Sensitivity of overhaul cost } \\
\hline 7 & 3 & 250 & 1000 & 2500 \\
\hline 8 & 3 & 250 & 1000 & 3500 \\
\hline
\end{tabular}

Table 4: Combination of cost parameters of sensitivity analysis

The results of the sensitivity analysis, presented in Table 5, highlight the consistency between the variation of each cost parameter, the optimal control factors $\left(Z p_{o}^{*}, n_{f}^{*}\right)$ and their respective incurred cost. The first observation from Table 5 is that the optimum control factors change in response to variations of the cost parameters. 


\begin{tabular}{|c|c|c|c|c|}
\hline Case & $Z p^{*}$ & $n_{f}^{*}$ & $\operatorname{Cost}^{*}$ & Remark \\
\hline Basic case & 8.79 & 13.15 & 146.35 & Base for the comparison \\
\hline \multicolumn{5}{|c|}{ Sensitivity of positive inventory cost } \\
\hline 1 & 9.95 & 12.50 & 136.03 & $Z p^{*}$ increases and $n_{f}^{*}$ decreases \\
\hline 2 & 7.66 & 13.84 & 155.29 & $Z p^{*}$ decreases and $n_{f}^{*}$ increases \\
\hline \multicolumn{5}{|c|}{ Sensitivity of backlog stock cost } \\
\hline 3 & 6.60 & 15.97 & 140.28 & $Z p^{*}$ decreases and $n_{f}^{*}$ increases \\
\hline 4 & 9.73 & 12.03 & 150.16 & $Z p^{*}$ increases and $n_{f}^{*}$ decreases \\
\hline \multicolumn{5}{|c|}{ Sensitivity of repair cost } \\
\hline 5 & 8.83 & 13.71 & 102.21 & $Z p^{*}$ almost unchanged and $n_{f}^{*}$ increases \\
\hline 6 & 8.74 & 12.50 & 190.33 & $Z p^{*}$ almost unchanged and $n_{f}^{*}$ decreases \\
\hline \multicolumn{5}{|c|}{ Sensitivity of overhaul cost } \\
\hline 7 & 8.74 & 12.58 & 143.26 & $Z p^{*}$ almost unchanged and $n_{f}^{*}$ decreases \\
\hline 8 & 8.83 & 13.65 & 149.31 & $Z p^{*}$ almost unchanged and $n_{f}^{*}$ increases \\
\hline
\end{tabular}

Table 5: Sensitivity analysis of different cost parameters

The variation of each cost, the respective control factors and optimal cost are examined and analyzed as follows:

Variation of the inventory cost, $\mathrm{C}^{+}$(cases 1 and 2): From the results obtained and presented in Table 5, we observe that the effect of the inventory cost is remarkable on the production threshold. For instance, with an increasing $c^{+}$(case 2), the production threshold $Z p^{*}$ decreases, because with higher inventory cost, the stock of product is more greatly penalized, leading to smaller production thresholds. The effect of this cost on the overhaul policy is such that when the inventory cost increases, the number of failures $n_{f}^{*}$ needed to perform the overhaul also increases, because the production threshold is reduced and the intersection with the overhaul zone moves to a higher number of failures. The final result of this condition is a reduction in the feasible overhaul zone $A^{\prime}$, (please see Figure 4 for an explanation of this reduction). The influence of the inventory cost also is remarkable in the incurred cost, as we observe that the more the inventory cost increases, the more the incurred cost rises as well. An opposite effect is observed on the production and overhaul policies at decreasing $c^{+}$(case 1).

Variation of the backlog cost, $\mathrm{C}^{-}$(cases 3 and 4): The backlog cost influences the production policy, and we observe that at increasing $c^{-}$(case 4), the production threshold increases, since, there is more wiggle room to maintain a certain amount of stock, and the increase in the inventory helps protect the system against shortages caused by breakdowns and defectives. With respect to the overhaul policy, the backlog cost also has an effect on its control parameter, since as the backlog cost increases, the production threshold increases, intersecting the overhaul zone at an early number of failures, thus leading to decrease $n_{f}^{*}$. This in turn yields to an increase in the feasible overhaul zone $A^{\prime}$. Decreasing $c^{-}$(case 3) produces the opposite result in both policies. Furthermore, as can be seen in the results obtained, the effect of the backlog cost on the control parameters is the opposite of the inventory cost effect. 
Variation of the repair cost, $c_{r}$ (cases 5 and 6): With respect to the repair cost, the influence of its variation shows that it does not considerably modify the optimal production threshold $Z p^{*}$, since this control parameter remained almost at the same level for the analyzed cases. The effect of the repair cost is observed mainly in the overhaul policy, where the underlying pattern shows, that as expected, when the $c_{r}$ increases (case 6), the number of failures $n_{f}^{*}$ decreases, thus implying an increase in the feasible overhaul zone $A^{\prime}$ with respect to the case with a low repair cost. Moreover, at decreasing $c_{r}$ (case 5), the overhaul zone $A^{\prime}$ decreases as well.

Variation of the overhaul cost, $c_{o}$ (cases 7 and 8): The main effect of this cost parameter is observed on the overhaul policy, since the production threshold remains almost at the same value for the analyzed cases. At decreasing $c_{o}$ (case 7), more overhauls are conducted, leading to a decrease in the number of repairs $n_{f}^{*}$. Moreover, at increasing $c_{o}$ (case 8), the overhaul zone $A^{\prime}$ is reduced, consequently leading to fewer overhauls and an increase in the number of repairs $n_{f}^{*}$. We notice that the effect of the overhaul cost on the overhaul policy is the opposite of the effect of the repair cost.

From the set of numerical examples considered in this sensitivity analysis, it is clear that the results obtained are logical and confirm the structure of our control policy. Generally, any cost variation reflects changes in the control parameters, and we observe that the inventory cost has the opposite effect as the backlog cost, while the repair cost has the opposite effect as the overhaul cost.

\subsection{Effect of the trajectory of the rate of defectives}

In the last section, we discussed the effect of variations of the cost parameters on the control factors and on the total incurred cost. The objective of this section is to allow a better understanding of the control parameters $\left(Z p_{o}, n_{f}\right)$ when varying the trajectory of the rate of defectives. Hence, in this section, another set of simulation runs were conducted in order to analyze the impact of variations of the trajectory of $\beta$ on the control factors. This analysis involves the variation of the parameter $r$ in expression (2). The obtained production thresholds $Z p_{o}^{*}$ and the number of failures $n_{f}^{*}$, are illustrated in Figures $11 \mathrm{a}$ and $11 \mathrm{~b}$, respectively, when varying the parameter $r$ from 0.25 to 3 , and the other parameters remain unchanged, as defined in the numerical values of the basic case.

The key observation in this analysis is that the role of the parameter $r$ in expression (2) is to adequately adjust the trajectory of the rate of defectives to a specific manufacturing system, as discussed previously in section 1, and presented graphically in Figure 2. An important remark should be made at this point, namely, that changes in the optimal control parameters $\left(Z p_{o}^{*}, n_{f}^{*}\right)$ appear when the path of the rate of defectives does not follow a linear trend. For instance, if the parameter is $r<1$, then the rate of defectives increases more abruptly, compared to the basic case $r=1$, and this condition results in a decrease in the number of failures $n_{f}^{*}$, consequently leading to more overhauls, as presented in Figure 11a. Additionally, when $r<1$, the production threshold increases, as depicted in Figure 11b, because the rate of defectives increases more rapidly, and so the 
production system needs more protection to cope with the defective products. Therefore, if the stock level increases and the overhaul is more conducted, then the total cost increases when $r<1$, because there is more disruptions caused by defectives.

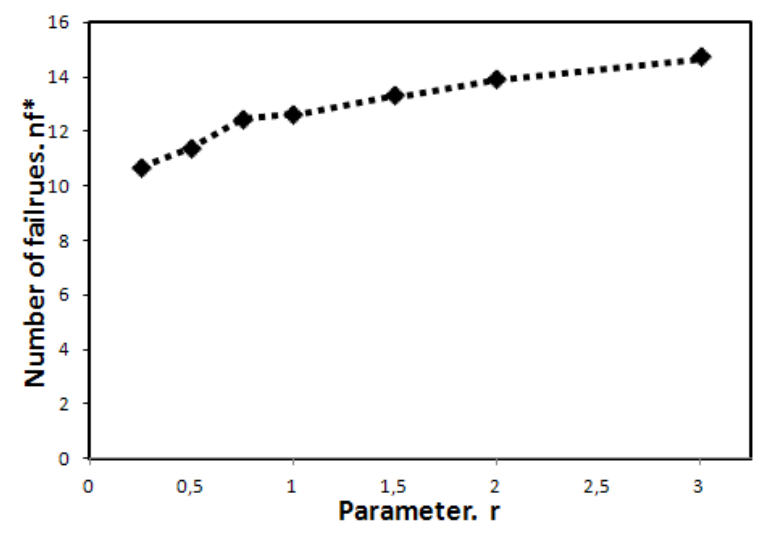

a) Number of failures, $\boldsymbol{n}_{f}^{*}$

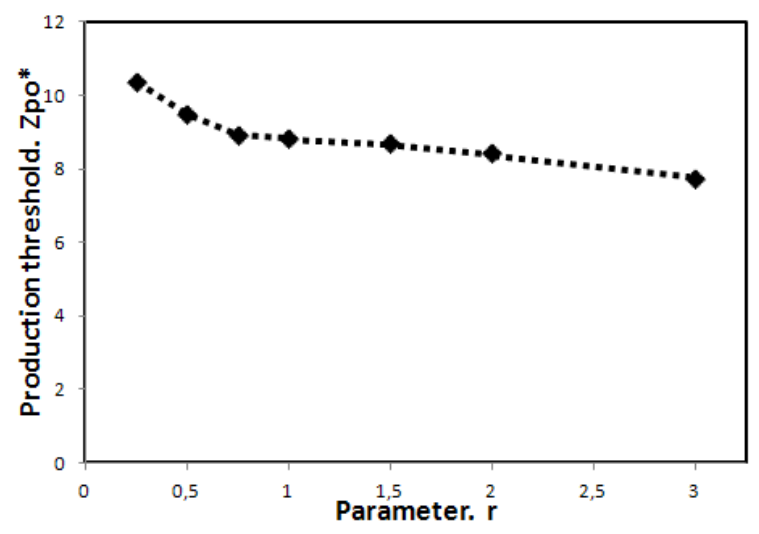

b) Production threshold, $Z p_{o}^{*}$

Figure 11: Effect of the parameter $r$ on the control parameters

There is another interesting observation when the parameter is $r>1$; it means that the rate of defectives increases more smoothly and slowly compared to the basic case, as can be seen in Figure 2. From the obtained results presented in Figure 11a, it follows that when $r>1$, the number of failures $n_{f}^{*}$ increases because the overhaul is conducted less frequently, reducing the feasible overhaul zone $A^{\prime}$. Furthermore, when the parameter is $r>1$, this leads to a reduction in the production threshold, since the rate of defectives smoothes its increase and thus reducing the need to maintain a certain amount of products as protection for shortages. With respect to the total cost, when $r>1$ it implies less defectives, leading to a reduction in the stock level and less overhaul conduction, thus the total cost decreases.

Through this sensitivity analysis, it is apparent that the variation of the trajectory of the rate of defectives has a clear effect on the control parameters and consequently in the total cost. In fact, we observed that when the parameter $r$ is less than one, the rate of defectives increases more abruptly, increasing the production threshold and reducing the number of failures $n_{f}^{*}$, leading to perform more overhauls, thus increasing the total cost. The opposite effect was observed, when the parameter $r$ is greater than one. From our results, we observed that the variation of the total cost given by changes of the parameter $r$ is around $\pm 10 \%$ of the total cost of the basic case, when $r=1$.

\section{Conclusions}

In this paper, issues pertaining to the inter-relation between production control and quality aspects are investigated in a manufacturing system consisting of an unreliable 
single machine, single part type manufacturing system. The machine is subject to deteriorations that have a negative effect on the quality of the parts produced, a fact that is especially reflected in the rate of defectives. The quality issues are related with the overhaul strategy, since it counters the effect of the deterioration. A simulation optimization approach is proposed and combines analytical formulation, simulation modeling, statistical analysis and response surface methodology. First, we investigate the structure of the joint production and overhaul control policy through the application of numerical methods designed for stochastic optimal control models. The resulting policy consists of a Machine Deterioration Dependent Hedging Point Policy which controls the production rate, and comprises several increasing thresholds and an overhaul strategy that performs a perfect repair when the number of failures is high. This leads to the identification of the two associated control parameters of the joint control policy as dependent on the deterioration of the production system. A simulation model is developed to reproduce the dynamic of the manufacturing system controlled by a modification of the hedging point policy obtained. An experimental design is applied to investigate the effects of the control factors on the incurred cost over the production horizon, and a cost function is estimated with a response surface from which we determine the best values of the control parameters and their respective incurred costs. The simulation optimization approach is applied on a wide range of time, and a combination of cost parameters is examined in a sensitivity analysis to evaluate their effects on the control policy. It is observed that in general, cost variations reflect changes in the control parameters and that the inventory cost has the opposite effect of the backlog cost, while the repair cost has the opposite effect of the overhaul cost. Also the effect of the variation of the trajectory of the rate of defectives is analyzed, and we observe that when the parameter $r$ is less than one, the rate of defectives increases more rapidly leading to an increase in the production threshold and simultaneously to more overhauls, thus reducing the number of failures $n_{f}^{*}$. The opposite effect on the control parameters is observed when the parameter $r$ is greater than one. The results obtained indicate that the control policy is influenced by the quality deterioration phenomenon, since in this case the hedging point policy is modified to a situation with several thresholds, which increase from one breakdown to the next. Hence, the production threshold increases with the number of failures, and the overhaul is conducted only with high numbers of failures. The use of equation (15) and its variations significantly reduces the number of runs needed in the simulation optimization. Finally, our control approach turned out as an interesting alternative for controlling the manufacturing system at the operational level, and the final assessment is that our simulation optimization approach is robust and efficient since it provides accurate results. 


\section{Appendix A. Optimality conditions}

The model denoted in equations (1-10) is a stochastic dynamic programming problem because the minimization operation takes into account the randomness of $\alpha$. Hence, we define the related transition rates $\lambda_{\mathrm{ij}}$ from modes $\mathrm{i}$ to $\mathrm{j}$, as follows:

$$
\operatorname{prob}[\alpha(t+\delta t)=i \mid \alpha(t)=j]=\lambda_{\mathrm{ij}} \delta t, \quad \forall \mathrm{i}, \mathrm{j}, \mathrm{i} \neq \mathrm{j}
$$

The value function $V(\alpha, x, n)$, defined in equations (10), denotes the value of the cost function (9) when the optimal control policy is applied. Regarding the principle of optimality, we can derive the optimality conditions of the problem; for example, let us assume we know the best possible trajectory during the time interval $[t, \infty]$. However, we know nothing about the problem during the interval $[0, t]$. If $V(\cdot, t)$ denotes a cost-to-go function at time $t$, then we can break up equation (10) as follows:

$V(\alpha(0), x(0), n(0), 0)=$

$$
\inf _{\substack{u(t), v(t) \\
0 \leq t \leq \infty}} E\left\{\begin{array}{c}
\int_{0}^{t} e^{-\rho t} g[(\alpha(t), x(t), n(t), u(t), v(t)] d t \\
+
\end{array} \mid \alpha(0), x(0), n(0)\right\}
$$

However, we know that the integral in the interval $[t, \infty]$ is the value function $V[\alpha(t), x(t), n(t), t]$, so considering the discounted rate $\rho$, equation (A.2) becomes:

$$
\begin{aligned}
& V(\alpha(0), x(0), n(0), 0)= \\
& \inf _{\substack{u(t), v(t) \\
0 \leq t \leq \infty}} E\left\{\begin{array}{c}
\int_{0}^{t} e^{-\rho t} g[(\alpha(t), x(t), n(t), u(t), v(t)] d t \\
+ \\
\frac{1}{1+\rho \delta t} V[\alpha(t), x(t), n(t), t]
\end{array} \mid \alpha(0), x(0), n(0)\right\}
\end{aligned}
$$

Based on this underlying pattern, if we perturb $t$, we can focus on $V[\alpha(t), x(t), n(t), t]$ to get the one-step counterpart in the interval $[t, t+\delta t]$, as indicated in the following expression:

$V(\alpha(t), x(t), n(t), t)=$

$$
\inf _{\substack{u(s), v(s) \\
t \leq s \leq t+\delta t}} E\left\{\begin{array}{c}
\int_{t}^{t+\delta t} e^{-\rho t} g[(\alpha(s), x(s), n(s), u(s), v(s)] d s \\
+ \\
\frac{1}{1+\rho \delta t} V[\alpha(t+\delta t), x(t+\delta t), n(t+\delta t), t+\delta t]
\end{array} \mid \alpha(t), x(t), n(t)\right\}
$$

where $u(s)$ is treated as constant in the interval $t \leq s \leq t+\delta t$. Equations (A.4) can be simplified even more if we apply the conditional expectation operation $\widetilde{E}$ (i.e., for any function $H(\alpha), \widetilde{E} H(\alpha(t+\delta t))=E\{H(\alpha(t+\delta t)) \mid \alpha(t)\})$. Thus, the expectation $\widetilde{E}$ leads to: 
$V(\alpha(t), x(t), n(t), t)=$

$$
\left.\inf _{\substack{u(s), v(s) \\
t \leq s \leq t+\delta t}} \tilde{E}\left\{\begin{array}{c}
g[(\alpha(t), x(t), n(t), u(t), v(t)] \delta t \\
+
\end{array}\right\} \begin{array}{c}
1 \\
\frac{1}{1+\rho \delta t} V[\alpha(t+\delta t), x(t+\delta t), n(t+\delta t), t+\delta t]
\end{array}\right\}+o(\delta t)
$$

Then for small $\delta t$ and after some manipulations, we have:

$$
\begin{aligned}
& \rho V(\alpha(t), x(t), n(t), t)= \\
& \inf _{u(t), v(t)} \tilde{E}\left\{\begin{array}{c}
g[(\alpha(t), x(t), n(t), u(t), v(t)] \delta t \\
+
\end{array}\right)+o(\delta t)
\end{aligned}
$$

The second term inside the expectation operator $\tilde{E}$ refers to the derivate of $V(\alpha, x, n)$. If the value function is differentiable, then we can apply the full derivative. Also, we can expand the expectation operation $\widetilde{E}$, (i.e., using the expansion $\widetilde{E} H(\alpha(t+\delta t))=H(\alpha(t))+$ $\sum_{j} H(j) \lambda_{j \alpha(\mathrm{t})} \delta t+o(\delta t)$, and so we have:

$$
\begin{aligned}
& \rho V(\alpha(t), x(t), n(t), t)= \\
& \inf _{u(t), v(t)}\left\{\begin{array}{c}
g[(\alpha(t), x(t), n(t), u(t), v(t)] \delta t+ \\
\frac{\partial V}{\partial x}[\alpha(t), x(t), n(t), t] \delta x(t)+\frac{\partial V}{\partial t}[\alpha(t), x(t), n(t), t] \delta t \\
+\sum_{\alpha^{\prime}} V\left[\alpha^{\prime}, x(t), n(t), t\right] \lambda_{\alpha^{\prime} \alpha(\mathrm{t})} \delta t
\end{array}\right\}+o(\delta t)
\end{aligned}
$$

We have eliminated the expectation symbol with the summation term. Now, if we replace $\delta x(t)$ by $\delta x(t)=\dot{x}(t) \delta t$, move $\frac{\partial V}{\partial t}$ to the left, let $\delta t$ approach zero, and do other manipulations, we get:

$$
\begin{aligned}
\rho V(\alpha, x, n, t)-\frac{\partial V}{\partial t}[\alpha, x, n, t] & = \\
\inf _{u(t), v(t)} & \left\{g[\alpha, x, n, u, v]+\frac{\partial V}{\partial x}[\alpha, x, n, t] \dot{x}+\sum_{\alpha^{\prime}} V\left[\alpha^{\prime}, x, n, t\right] \lambda_{\alpha^{\prime} \alpha}\right\}
\end{aligned}
$$

We observe that none of the functions $g(\cdot)$ and $\dot{x}(\cdot)$ are functions of $t$ explicitly. Furthermore, since the time horizon is infinite and a steady-state distribution exists for $\alpha$, equation (A.8) is independent of $t$. Based on this, and replacing the summation term by the generator $\mathrm{Q}(\cdot)=\left\{\lambda_{\alpha \alpha^{\prime}}(\cdot)\right\}$, equations (A.8) can be further simplified to:

$$
\rho V(\alpha, x, n)=\inf _{(u, v) \in \Gamma(\alpha)}\left\{g[\alpha, x, n, u, v]+\frac{\partial V}{\partial x}[\alpha, x, n] \dot{x}+Q(\cdot) V[\alpha, x, \varphi(\xi, n)](\alpha)\right\}
$$

These are the fundamental manufacturing system control equations called Hamilton-Jacobi-Bellman (HJB) equations, and they are important because they convert the minimization problem, defined over an extended time interval, into a minimization problem at a single time instant. HJB equations serve us to determine the optimal control policy, which in this case, is a real feedback law. Since the problem is stochastic, it means that it is specified only when $(\alpha, x, n)$ are determined. Further details about how HJB equations are obtained can be consulted in Rishel (1975) and Gershwin (2002). 


\section{References}

[1] Akella, R. and Kumar, P.R., 1986, Optimal Control of Production Rate in a Failure Prone Manufacturing System, IEE Transactions on Automatic Control, Vol. AC-31m No. 2, pp. 116-126.

[2] Berthaut, F., Gharbi, A., Kenné, J.P., Boulet, J.F., 2010, Improved joint preventive maintenance and hedging point policy, International Journal of Production Economics, No. 127, pp. 60-72.

[3] Boschian, V., Rezg, N., Chelbi, A., 2009, Contribution of simulation to the optimization of maintenance strategies for a randomly failing production system, European Journal of Operational Research, No. 197, pp. 1142-1149.

[4] Boukas, E.K. and Hauire, A., 1990, Manufacturing flow control and preventive maintenance: a stochastic control approach, IEEE Transactions on Automatic Control 33, pp. 1024-1031.

[5] Chan, F.T.S., Wang, Z., Zhang, J., Wadhwa, S., 2008, Two-level hedging point control of a manufacturing system with multiple product-types and uncertain demands, International Journal of Production Research, 46:12, pp. 3259-3295.

[6] Colledani, M. and Tolio, T., 2006, Impact of Quality Control on Production System Performance, CIRP Annals - Manufacturing Technology, Vol. 55, No. 1, pp. 453-456.

[7] Colledani, M. and Tolio, T., 2009, Performance evaluation of production systems monitored by statistical process control and off-line inspections, International Journal of Production Economics, 120, pp. 348-367.

[8] Colledani, M. and Tolio, T., 2011, Integrated analysis of quality and production logistics performance in manufacturing lines, International Journal of Production Research, 49:2, pp. 485-518.

[9] Dehayem Nodem F.I., Kenné, J.P., Gharbi, A., 2011, Simultaneous control of production, repair/replacement and preventive maintenance of deteriorating manufacturing systems, International Journal of Production Economics, No. 134, pp. 271-282.

[10] Dhouib, K., Gharbi, A., Ben Aziza, M.N., 2012, Joint optimal production control/preventive maintenance policy for imperfect process manufacturing cell, International Journal of Production Economics 137, pp. 126-136.

[11] Gershwin, S.B., 2002, Manufacturing Systems Engineering, Massachusetts Institute of Technology, Second private printing, Cambridge, Massachusetts, US., 501 p. 
[12] Gharbi, A. and Kenné, J.P., 2000, Production and preventive maintenance rates control for a manufacturing system: An experimental design approach, International Journal of Production Economics, 65, pp. 275-287.

[13] Gharbi, A., Hajji, A., Dhouib, K., 2011, Production rate control of an unreliable manufacturing cell with adjustable capacity, International Journal of Production Research, 49:21, pp. 6539-6557.

[14] Hajji, A., Gharbi, A., Kenné, J.P., 2009, Joint replenishment and manufacturing activities control in two stages unreliable supply chain. International Journal of Production Research 47 (12), pp. 3231-3251.

[15] Hajji, A., Mhada F., Gharbi, A., Pellerin, R., Malhame, R., 2011, Integrated product specifications and productivity decision making in unreliable manufacturing systems, International Journal of Production Economics, 129, pp. $32-42$.

[16] Huang, D., Allen, T.T., Notz, W.I., and Zeng, N., 2006, Global Optimization of Stochastic Black-Box Systems via Sequential Kriging Meta-Models, Journal of Global Optimization, vol. 34, pp. 441-466.

[17] Inman, R.R., Blumenfeld D.E., Huang, N., 2003, Designing production systems for quality: research opportunities from an automotive industry perspective, International Journal of Production Research, 41:9, 1953-1971.

[18] Kenné, J.P., Boukas, E.K., Gharbi, A, 2003, Control of production and corrective maintenance rates in a multiple-machine, multiple-product manufacturing system, Mathematical and Computer modeling 38 (3-4), pp. 351-365.

[19] Kenné, J.P., Dejax, P., Gharbi, A., 2012, Production planning of a hybrid manufacturing-remanufacturing system under uncertainty within a closed-loop chain, International Journal of Production Economics 135, pp. 81-93.

[20] Kim, J. and Gershwin, S.B., 2005, Integrated quality and quantity modeling of a production line, OR Spectrum, 27, pp. 287-314.

[21] Kim, J. and Gershwin, S.B., 2008, Analysis of long flow lines with quality and operational failures, IIE Transactions, 40, pp. 284-296.

[22] Kimemia, J.G, and Gershwin, S.B, 1983, An algorithm for the computer control of production in a flexible manufacturing system, IIE Transactions, 15(4), pp. 353-362.

[23] Kushner, H.J. and Dupuis, P.G., 1992, Numerical Methods for Stochastic Control Problems in Continuous Time, (Springer, New York, NY).

[24] Lam, Y., Zhu, L.X., Chan, J.S.K., Liu, Q., 2004, Analysis of data from a series of events by a geometric process model, Acta Mathematicae Applicatae 20, pp. 263-282. 
[25] Lam, Y., 2007, A geometric process maintenance model with preventive repair, European Journal of Operation Research, 182, pp. 806-819.

[26] Lavoie, P., Gharbi, A, Kenné, J.P., 2009a, A comparative study of pull control mechanisms for unreliable homogenous transfer lines, International Journal of Production Economics, doi:10.1016/j.ijpe.2009.11.022.

[27] Lavoie, P., Gharbi, A., Kenné, J.P., 2009b, Optimization of production control policies in failure-prone homogenous transfer lines, IIE Transactions, 41:3, pp. 209-222.

[28] Mhada, F., Hajji, A., Malhame, R., Gharbi, A., Pellerin, R., 2011, Production control of unreliable manufacturing systems producing defective items, Journal of Quality in Maintenance Engineering, Vol. 17, No. 3, pp. 238-253.

[29] Montgomery, D.C., 2009, Design and Analysis of Experiments, John Wiley \& Sons, Inc., Seven Edition, US.

[30] Mourani, I., Hennequin, S., Xie, X., 2008, Simulation-based optimization of a single-stage failure-prone manufacturing system with transportation delay, International Journal of Production Economics, 112, pp. 26-36.

[31] Njike, A.N., Pellerin, R., Kenné, J.P., 2009, Simultaneous control of maintenance and production rates of a manufacturing system with defective products, Journal of Intelligent Manufacturing 10845, article 354.

[32] Pham, H. and Wang H., 1996, Imperfect maintenance, European Journal of Operation Research, No. 94, pp. 425-438.

[33] Radhoui, M., Rezg, N., Chelbi, A., 2009, Integrated model of preventive maintenance, quality control and buffer sizing for unreliable and imperfect production systems, International Journal of Production Research, Vol. 47, No. 2, pp. 389-402.

[34] Wang, H., 2002, A survey of maintenance policies of deteriorating systems, European Journal of Operation Research, No. 139, pp. 468-489.

[35] Wang, H. and Pham, H., 1999, Some maintenance models and availability with imperfect maintenance in production systems, Annual of Operation Research No. 91, pp. 305-318. 